\title{
Hysteresis loop area scaling exponents in DNA unzipping by a periodic force: A Langevin dynamics simulation study
}

\author{
Rajeev Kapr* \\ Department of Physical Sciences, Indian Institute of Science Education and Research Mohali, \\ Sector 81, Knowledge City, S. A. S. Nagar, Manauli PO 140306, India.
}

(Dated: August 4, 2021)

\begin{abstract}
Using Langevin dynamics simulations, we study the hysteresis in unzipping of longer doublestranded DNA chains whose ends are subjected to a time-dependent periodic force with frequency $\omega$ and amplitude $G$ keeping the other end fixed. We find that the area of the hysteresis loop, $A_{l o o p}$, scales as $1 / \omega$ at higher frequencies, whereas it scales as $\left(G-G_{c}\right)^{\alpha} \omega^{\beta}$ with exponents $\alpha=1$ and $\beta=1.25$ in the low-frequency regime. These values are same as the exponents obtained in Monte Carlo simulation studies of a directed self-avoiding walk model of a homopolymer DNA [R. Kapri, Phys. Rev. E 90, 062719 (2014)], and the block copolymer DNA [R. K. Yadav and R. Kapri, Phys. Rev. E 103, 012413 (2021)] on a square lattice, and differs from the values reported earlier using Langevin dynamics simulation studies on a much shorter DNA hairpins.
\end{abstract}

\section{INTRODUCTION}

The unzipping of a double stranded DNA (dsDNA) by a mechanical force, which is an initial step in biological processes like DNA replication and RNA transcription [1, has been studied over two decades both theoretically 2,8 and experimentally using single-molecule manipulation techniques 9 -13. The two strands of a dsDNA, whose ends are exerted by a pulling force, is found to unzip to two single strands if the force exceeds a critical value. The unzipping transition, which is now well established, is a first-order phase transition. If the externally applied force is oscillatory in nature, then it unzips and rezips the two strands of the DNA in each cycle, and the force-extension isotherm shows a hysteresis. There have been many studies of hysteresis in unbinding and rebinding of biomolecules in recent years because it reveals important information about the kinetics of conformational transformations, the potential energy landscape, and controlling the folding pathway of a single molecule and in force sensor studies 13 17.

In recent years, the behavior of a dsDNA under a periodic force with frequency $\omega$ and amplitude $G$ has been studied by using Brownian dynamics (BD) or Langevin dynamics (LD) simulation of an off-lattice coarse-grained model for short chains which are limited to a maximum number of $N=16$ base pairs and 32 monomers [1822], and by using Monte Carlo (MC) simulations of DNA chains having 1024 monomers with $N=512$ base pairs on a $(D=1+1)$-dimensional square lattice 2326$]$. Both $\mathrm{LD}$ and MC simulation studies show the existence of a dynamical phase transition, where the DNA can be taken from the zipped state to an unzipped state via a new dynamical state. The area of the hysteresis loop, $A_{\text {loop }}$, which represents the energy dissipated in the system, is found to scale as $1 / \omega$ at higher frequencies, whereas at low frequencies, $A_{\text {loop }}$ scales as $G^{\alpha} \omega^{\beta}$, or $\left(G-G_{c}\right)^{\alpha} \omega^{\beta}$,

\footnotetext{
* rkapri@iisermohali.ac.in
}

where $G_{c}$ is the critical force needed to unzip the DNA for the static force case. The values of the exponents $\alpha$ and $\beta$ obtained in $\mathrm{BD} / \mathrm{LD}$ and $\mathrm{MC}$ simulations are, however, different. In $\mathrm{BD} / \mathrm{LD}$ simulation studies on shorter DNA hairpins [18 22, a chain having $2 N$ monomers, whose first $N$ monomers are complementary to the rest half. The monomers of the chain are chosen in such a manner that the $i$ th monomer from the anchored end can bind only with the $(N-i)$ th monomer of the chain, thus mimicking a base pair of the DNA. One end of the chain is anchored at the origin and an external time dependent periodic force $g(t)$ is applied on the free end along $x$ direction and its distance from the origin, $x(t)$, is monitored. In MC simulation studies [23 26], the strands of DNA are represented by two directed self-avoiding walks (DSAWs), which do not cross each other, on a $(D=1+1)$-dimensional square lattice. Whenever the $i$ th monomers of walks (mimicking complementary bases) are unit distance apart, there is a gain in energy (base pairing). Two strands of the DNA at one end are always kept fixed at origins $O$ and $O^{\prime}$ and the other end monomers are subjected to a time-dependent periodic force $g(t)$ and the separation, $x(t)$, between them is monitored. In both $\mathrm{BD} / \mathrm{LD}$ and $\mathrm{MC}$ simulation studies, the average forcedistance isotherms obtained from the time series show hysteresis loop whose area is studied as a function of $G$ and $\omega$. Initial $\mathrm{BD} / \mathrm{LD}$ simulation studies reported exponent values $\alpha=\beta=1 / 2[18-20$. These were later modified to $\alpha=0.33$ and $\beta=1 / 2$ [21]. However, a different set of exponents, $\alpha=1$ and $\beta=1.25$, were obtained for longer homopolymer DNA chains in MC simulation studies [24. Very recently, the DSAW model has been extended to study the unzipping of a block copolymer DNA subjected to a periodic force, and the same set of exponents $(\alpha=1$ and $\beta=1.25)$ were obtained [26]. This inspired us to perform LD simulation studies on a longer DNA chains and investigate the true values of the loop area exponents at low-frequency regime. Our hypothesis is that the different set of exponents obtained in earlier BD/LD studies, as compared to MC studies, are due to the presence of strong finite-size effects because of shorter 
chain lengths used in those studies.

In this paper, we study the unzipping transition of a dsDNA subjected to a periodic force using LD simulations in two dimensions (2D) and compare our results to a wellestablished DSAW model of a DNA on a $D=1+1$ square lattice. The later model has been studied extensively, for over two decades, using the generating function, exact transfer matrix, and Monte Carlo techniques [5] 7, 2329. In unzipping transition, the average of the relative distance $\mathbf{r}=\left|\mathbf{r}_{\mathbf{2}}(N)-\mathbf{r}_{\mathbf{1}}(N)\right|$ between the end monomers of the two strands of the DNA (the order parameter), which is the conjugate variable to an externally applied force, is always along the direction of the force. The fluctuations in the transverse directions are so small that they can be safely neglected. In the absence of any external pulling force, the two strands of the DNA can also be denatured thermally, purely due to the competition between the entropy and the energy, at a temperature $T_{M}$ known as the melting temperature of DNA. Unlike thermal melting, which depends on both the model and dimension used, the force-induced transition at $T<T_{M}$ was found to be independent of both the model and the dimension. The values of the critical force and the melting temperature are, however, model dependent [5, 6]. The length of the DNA simulated in this paper for the periodic case (up to 192 monomers with $N=96$ base pairs) are six times longer than the chain lengths used in earlier $\mathrm{BD} / \mathrm{LD}$ simulation studies $[18,22$. We first consider the static force case and confirm that the model considered in this paper indeed show first-order phase transition same as DSAW model. We obtain the value of the critical force, $G_{c}(T)$, needed to unzip the dsDNA at two different temperatures and also the melting temperature $T_{M}$ for the model. Next, we consider the periodic force case, where the force-distance isotherms show hysteresis loop whose area, $A_{\text {loop }}$, behaves nonmonotonically with the frequency. We observe that the loop area scales as $A_{\text {loop }} \sim 1 / \omega$ in the higher-frequency regime, whereas it scales as $A_{\text {loop }} \sim\left(G-G_{c}\right)^{\alpha} \omega^{\beta}$ with exponent values $\alpha \approx 1$ and $\beta \approx 1.25$ in the lower-frequency regime. These exponents are similar to the exponents obtained in earlier MC simulation studies on longer chain lengths [24, 26].

The paper is organized as follows: In Sec. III, we define the model simulated in this paper. The results are discussed in Sec. III and summarized in Sec. IV.

\section{MODEL}

We model the strands of a dsDNA by beads and springs in two dimensions (see Fig. 1). The beads of the polymer experience an excluded volume interaction modeled by the Weeks-Chandler-Andersen potential of the form

$$
U_{\text {bead }}(r)= \begin{cases}4 \varepsilon\left[\left(\frac{\sigma}{r}\right)^{12}-\left(\frac{\sigma}{r}\right)^{6}\right]+\varepsilon & \text { for } r \leq r_{\min } \\ 0 & \text { for } r>r_{\min }\end{cases}
$$

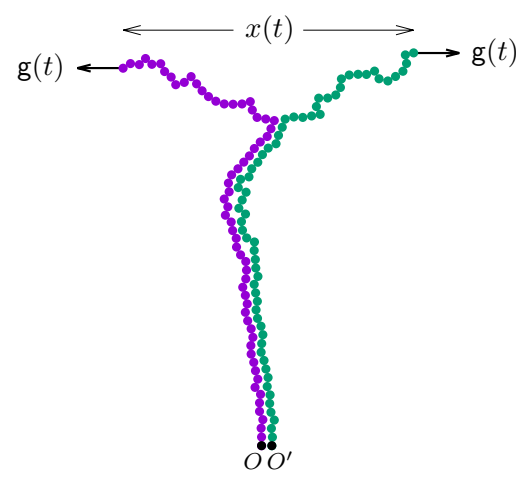

FIG. 1. Schematic diagram of a dsDNA. One end of the DNA is anchored at the origin $\left(O\right.$ and $\left.O^{\prime}\right)$ and the strands on the free end are subjected to a time-dependent periodic force $\mathrm{g}(t)$ with frequency $\omega$ and amplitude $G$.

where, $\varepsilon$ is the strength of the potential. The cutoff distance, $r_{\min }=2^{1 / 6} \sigma$, is set at the minimum of the potential. The consecutive monomers of strands are connected by the finitely extensible nonlinear elastic (FENE) potential [30] of the form

$$
U_{\mathrm{FENE}}(r)=-\frac{1}{2} k R_{0}^{2} \ln \left(1-\frac{r^{2}}{R_{0}^{2}}\right),
$$

where $k$ and $R_{0}$ are the spring constant and the maximum allowed distance between the consecutive monomers, respectively. The complementary monomers of the DNA (i.e., $i$ th monomers of both the strands) interacts with each other via standard LJ potential:

$$
U_{\mathrm{bp}}(r)= \begin{cases}4 \varepsilon_{\mathrm{p}}\left[\left(\frac{\sigma}{r}\right)^{12}-\left(\frac{\sigma}{r}\right)^{6}\right] & \text { for } r \leq r_{\mathrm{c}} \\ 0 & \text { for } r>r_{\mathrm{c}},\end{cases}
$$

where $\varepsilon_{\mathrm{p}}$ denotes the base pair interaction strength and $r_{\mathrm{c}}=2.5 \sigma$ is the cutoff distance.

The strands at one end of the DNA are anchored at $O$ and $O^{\prime}$, which are $1.12 \sigma$ distance apart, and the strands at the free end are subjected to a time-dependent periodic force

$$
\mathbf{g}(t)=G|\sin (\omega t)|,
$$

where $G$ is the amplitude and $\omega$ is the angular frequency of the oscillating force.

To integrate the equation of motion for the monomers of the chain we use LD algorithm with velocity-Verlet update. The equation of motion for a monomer is given by

$$
m \ddot{\boldsymbol{r}}_{i}=-\boldsymbol{\nabla} U_{i}+\mathrm{g}-\zeta \boldsymbol{v}_{i}+\boldsymbol{\eta}_{i},
$$

where $m$ is the monomer mass, $U_{i}=U_{\text {bead }}+U_{\text {FENE }}+U_{\mathrm{bp}}$ is the total potential experienced by $i$ th monomer, $\zeta$ is the friction coefficient, $\boldsymbol{v}_{i}$ is the monomer's velocity, 
and $\boldsymbol{\eta}_{i}$ is the random force satisfying the fluctuationdissipation theorem $\left\langle\eta_{i}(t) \eta_{j}\left(t^{\prime}\right)\right\rangle=2 \zeta k_{B} T \delta_{i j} \delta\left(t-t^{\prime}\right)$. The unit of energy, length, and mass are set by $\varepsilon$, $\sigma$, and $m$, respectively, which sets the unit of time as $\tau=\sqrt{m \sigma^{2} / \varepsilon}$. In these reduced units, we choose $\zeta=1.0$, $\varepsilon_{\mathrm{p}}=\varepsilon, k=30 \varepsilon / \sigma, R_{0}=1.5 \sigma$, and $k_{B} T=0.1 \varepsilon$. The force is measured in units of $\sigma / \varepsilon$. A time step of $\Delta t=0.005$ is used in all simulation runs. The simulations are done using LAMMPS software 31.

The distance between the end monomers of the two strands is monitored as a function of time, $x(t)$, for various force amplitudes $G$ and frequency $\omega$. Due to the periodic nature of the applied force, the extension $x(\mathrm{~g})$ as a function of force $\mathrm{g}$ can be obtained from the time series $x(t)$. This is then averaged over 1000 cycles to obtain the average extension, $\langle x(\mathrm{~g})\rangle$. For longer chains (i.e., $N=64$ and 96 ), the computation is very costly in the lower-frequency regime 33. However, it was observed that, in this regime, the averaging over even 100 cycles is good enough to give a smooth $x(\mathrm{~g})$ vs $\mathrm{g}$ loop. To be on a safer side, we have used 200 cycles for averaging after leaving the first 20 cycles for the system to reach the stationary state. For the force amplitude $G$ and the frequencies $\omega$ used in this work, the average extension, $\langle x(\mathrm{~g})\rangle$, for the forward and the backward paths is not the same and a hysteresis loop is observed. The area of the hysteresis loop, $A_{\text {loop }}$, defined as

$$
A_{\text {loop }}=\oint\langle x(\mathrm{~g})\rangle d \mathrm{~g},
$$

depends on the frequency $\omega$ and the amplitude $G$ of the periodic force and serves as a dynamical order parameter [32. The area of the loop, $A_{\text {loop }}$ is obtained numerically using the trapezoidal rule after dividing the interval $\mathrm{g} \in[0, G]$ into $10^{5}$ equally spaced intervals, and interpolating the value of $\langle x(\mathrm{~g})\rangle$ at the ends of these intervals using cubic splines of GNU Scientific Library [34.

\section{RESULTS AND DISCUSSIONS}

\section{A. Static case}

Let us first consider the equilibrium case where the dsDNA is subjected to a constant pulling force, i.e., $\mathrm{g}(t)=G$ and check whether the average separation behaves similarly as that obtained from the DSAW model.

In Figs. 2(a) and 2(b), we have plotted the average separation between the strands of the DNA, where a constant pulling force $G$ is acting, at various $G$ values for DNA having $N=64,96,128,192$, and 256 base pairs at temperatures $T=0.1$ and $T=0.2$, respectively. For smaller values of the force, the average separation, $\langle x(G)\rangle$, which acts as an order parameter, is zero showing that the two strands of the DNA are in the zipped phase. On increasing the force value, the average separation abruptly increases at some critical force value, $G_{c}(T)$, which depends on the temperature, and $\langle x(G)\rangle \sim N$ showing that the DNA is in the unzipped phase. The critical value of force $G_{c}(T)$, can be obtained by using the finite-size scaling (FSS) of the form

$$
\langle x(G)\rangle=N^{d} \mathcal{G}\left(\frac{\left(G-G_{c}\right)}{N^{\phi}}\right),
$$

where $d$ and $\phi$ are the critical exponents. In Figs. 2(c) and 2(d), we have plotted the scaled separation $\langle x(G)\rangle / N^{d}$ for the DNA of various chain lengths as a function of $\left(G-G_{c}\right) / N^{\phi}$ at $T=0.1$ and $T=0.2$, respectively. The data for various chain lengths collapse on a scaling curve for the set of critical exponents $d=1.10 \pm 0.05, \phi=1.00 \pm 0.05$ with critical force value $G_{c}(T=0.1)=0.45 \pm 0.05$ for $T=0.1$, and exponents $d=1.05 \pm 0.05, \phi=1.00 \pm 0.05$ with $G_{c}(T=0.2)=0.25 \pm 0.05$ for $T=0.2$. The unzipping exponents $d=1$ and $\phi=1$, which are same as the exponents obtained for the $D=1+1$ case, show that the scaled mean separation between the strands behave, in the thermodynamic limit (i.e., $N \rightarrow \infty$ ), as

$$
\langle x(G)\rangle / N \sim\left\{\begin{array}{l}
X_{-} \text {for } G<G_{c}(T) \\
X_{+} \text {for } G>G_{c}(T)
\end{array}\right.
$$

i.e., having two different values with a jump discontinuity at $G_{c}(T)$, implying the first-order nature of the unzipping transition [2, 5, 7].

In the absence of a pulling force, the free ends of the DNA can move freely in both the $x$ and $y$ directions and perform more like self-avoiding walks (SAWs). Let $\left(x_{1}, y_{1}\right)$ and $\left(x_{2}, y_{2}\right)$ represent the coordinates of the end monomers of two strands of the DNA. The distance between the end monomers can then be obtained by $\mathrm{r}=\sqrt{\left(x_{2}-x_{1}\right)^{2}+\left(y_{2}-y_{1}\right)^{2}}$. In Fig. 3(a), we have plotted the average separation $\langle\mathrm{r}\rangle$ as a function of temperature $T$ for the DNA of various lengths $N=64,96,128$, 192, and 256. To estimate the melting temperature $T_{M}$ of the DNA, we use the FSS of the form

$$
\langle\mathrm{r}\rangle \sim N^{d_{t}} \mathcal{Y}\left(\left(T-T_{M}\right) N^{\phi_{t}}\right),
$$

where $d_{t}$ and $\phi_{t}$ are the critical exponents for the denaturation transition. When the scaled separation $\langle\mathrm{r}\rangle / N^{d_{t}}$ for various chain lengths are plotted as a function of $\left(T-T_{M}\right) N^{\phi_{t}}$, a nice collapse is obtained for the exponent values $d_{t}=0.75 \pm 0.05, \phi_{t}=1.00 \pm 0.05$ and $T_{M}=0.30 \pm 0.01$ [see Fig.. 3(b)]. The melting of a dsDNA is a continuous transition in our model. Note that, at $T \geq T_{M}$, the exponent $d_{t}$ depends on dimensions as expected. In $2 \mathrm{D}$, the value $d_{t}=0.75$ is consistent with the size exponent $\nu=3 / 4$ of a SAW in $2 \mathrm{D}$ [35], whereas, in $D=1+1$, the end separation performs a random walk in $1 \mathrm{D}$ and the exponent $d_{t}=0.5$ (see, e.g., Ref. [26]) is consistent with the size exponent $\nu=1 / 2$ of a random walker [35].

Once the melting temperature $T_{M}$ for the model is obtained, it is easy to estimate the characteristic hydrogen bond energy $\varepsilon$ in real units and compare our results with 

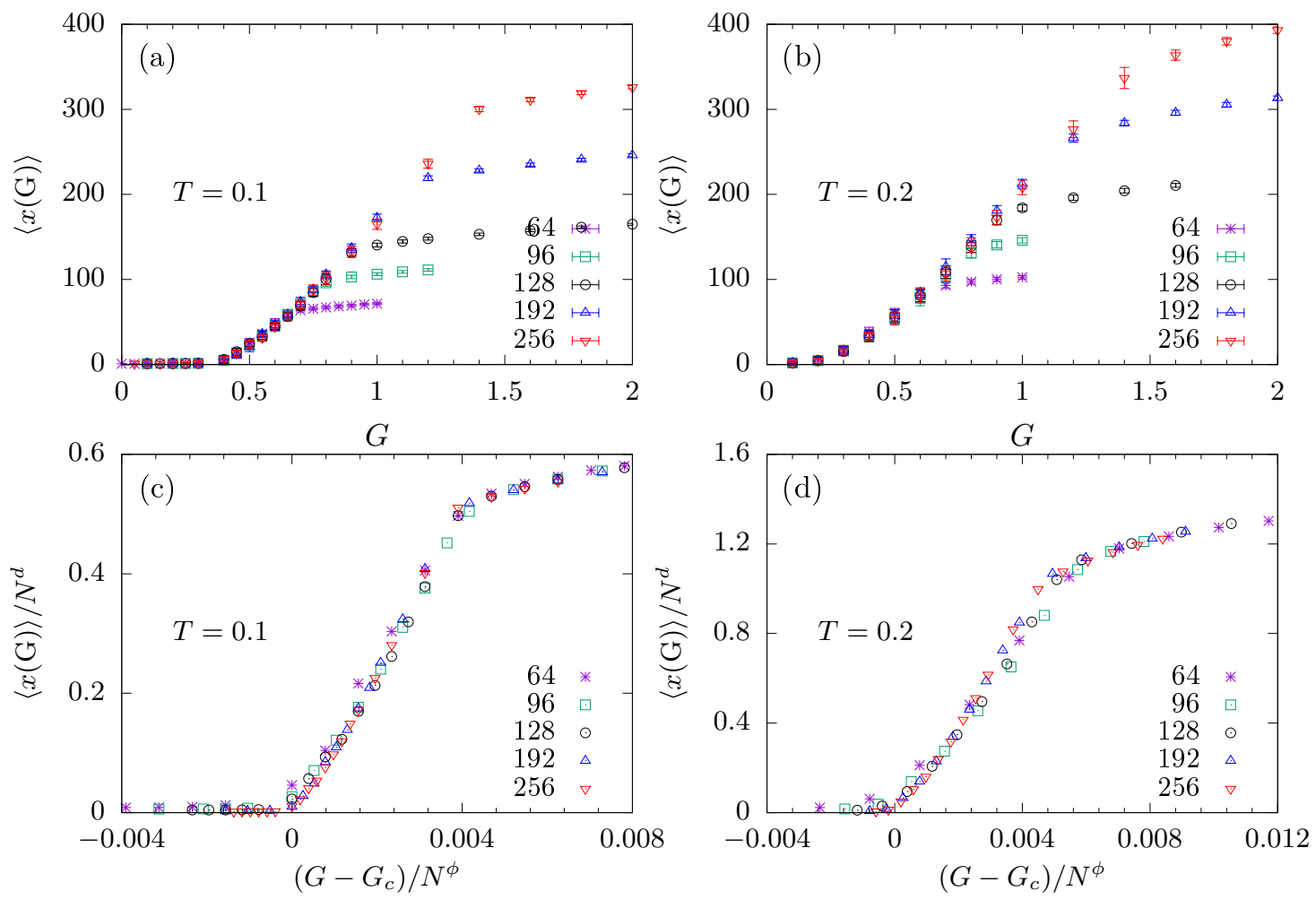

FIG. 2. Average separation $\langle x(\mathrm{G})\rangle$ between the free strands of the DNA as a function of pulling force $G$ for various chain lengths $N=64,96,128,192$, and 256 at (a) $T=0.1$ and (b) $T=0.2$. Plot of scaled separation $\langle x(G)\rangle / N^{d}$ vs $\left(G-G_{c}\right) / N^{\phi}$ showing a nice collapse for (c) $T=0.1$ with exponents $d=1.10 \pm 0.05, \phi=1.00 \pm 0.05$ and critical force $G_{c}(T=0.1)=0.45 \pm 0.05$ and (d) for $T=0.2$ with exponents $d=1.05 \pm 0.05, \phi=1.00 \pm 0.05$, and critical force $G_{c}(T=0.2)=0.25 \pm 0.05$.

the unzipping experiments. If $T_{M}^{*}$ represents the melting temperature in real units, then it is related to $T_{M}$ by $T_{M}=k_{B} T_{M}^{*} / \varepsilon$. Using $T_{M}=0.3$, and $T_{M}^{*}=363$ $\mathrm{K}[11$, we obtain $\varepsilon \approx 0.1 \mathrm{eV}$. Considering $\sigma=5.17 \AA$ as the distance at which the interparticle potential between the base pairs goes to zero, and $m=5 \times 10^{-22} \mathrm{~g}$ as the average mass of each monomer, the unit of time is obtained as $\tau=\sqrt{m \sigma^{2} / \varepsilon} \approx 3$ ps [see Ref. 19] and references therein]. The time and the distances are measured in real units as $t^{*}=\tau t$ and $r^{*}=\sigma r$, respectively. The order of the force is given by $\sigma / \AA \sim 160 \mathrm{pN}$. Using similar arguments as in Ref. [19, the temperature conversion formula to real units below the melting temperature for our model can be obtained as $T^{*}=363+280(T-0.30)$ $\mathrm{K}$. Therefore, the reduced temperature $T=0.1$ simulated in our paper corresponds to $307 \mathrm{~K}$ (i.e., $34^{\circ} \mathrm{C}$ ). The critical force $G c=0.45$ in reduced units corresponds to $G_{c}^{*} \approx 14 \mathrm{pN}$ similar to the critical force obtained in the experiments [11.

\section{B. Dynamic case}

From earlier studies, it is known that when a dsDNA is subjected to a periodic force it can be unzipped either by keeping the amplitude $G$ fixed and changing the frequency $\omega$ or vice versa. If $G$ is not very small, and $\omega$ is sufficiently high to avoid equilibration of the DNA, then we obtain a hysteresis loop for the average extension $\langle x(\mathrm{~g})\rangle$, whose area, $A_{\text {loop }}$, depends on $G$ and $\omega$. In Fig. 4(a), we have shown the behavior of $A_{\text {loop }}$ as a function of $\omega$ for the DNA of length $N=64$ for $G=1.5$ at $T=0.1$. The area of the loop increases with the frequency, reaches a maximum and then decreases as the frequency is increased further. The loops at four different frequencies, labeled by $P, Q, R$, and $S$ in Fig. 4 (a), are shown in Fig. 4(b). Since the force amplitude $G=1.5$ is about three times the critical force needed to unzip the DNA at $T=0.1$, the stationary state of the DNA is unzipped state. At a higher frequency $\omega_{P}=6.28 \times 10^{-3}$, the applied force fluctuates very rapidly and the DNA does not get time to respond to this change. As a result, the DNA remains in the unzipped state, as indicated by the higher values of the average extension, $\langle x\rangle$, with a small loop area. On decreasing the frequency to $\omega_{Q}=6.28 \times 10^{-4}$, the DNA still remains in the unzipped phase but with slightly increase in the loop area. On decreasing the frequency further to $\omega_{R}=6.28 \times 10^{-5}$, the DNA gets enough time to relax to the oscillating force. Therefore, during the portion of the cycle where the instantaneous force value is less than the critical force $G_{c}$, the two strands of the DNA come together and the com- 

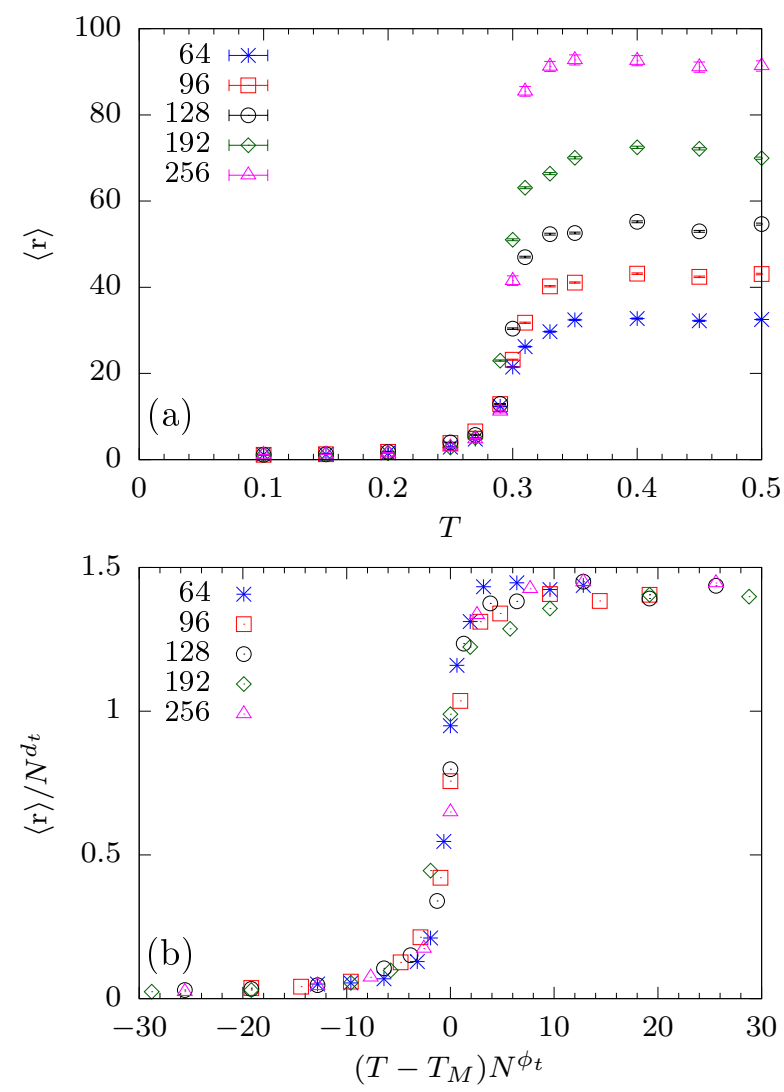

FIG. 3. (a) Average separation $\langle\mathrm{r}\rangle$ between the end monomers of two strands of the DNA as a function of temperature $T$ for various chain lengths. (b) Scaled separation $\langle\mathrm{r}\rangle / N^{d_{t}}$ vs $\left(T-T_{M}\right) N^{\phi_{t}}$ showing a nice collapse for exponents $d_{t}=0.75 \pm$ $0.05, \phi_{t}=1.00 \pm 0.05$, and the melting temperature $T_{M}=$ $0.30 \pm 0.01$.

plementary base pairs are formed, resulting the DNA in the zipped phase with a large hysteresis loop area. This is indicated by the lower values of the average extension, $\langle x\rangle$ for smaller g values in Fig. 4(b). On decreasing the frequency further to $\omega_{S}=6.28 \times 10^{-6}$, the two strands have ample time to relax in the lower as well as higher values of force thus resulting in a very small loop area in the transition region. This loop area will eventually go to zero on decreasing the frequency further.

In Fig. 5, we have plotted $A_{\text {loop }}$ as a function of $\omega$ at various force amplitudes $G$ for the DNA of length $N=64$. The figure shows that the frequency, $\omega^{*}(G)$, at which the loop area is maximum depends on the amplitude $G$ of the oscillating force. We observe that for smaller $G$ values, the $A_{\text {loop }}$ curves have broader peaks. The peak becomes narrower with the increase in the force amplitude. Furthermore, on increasing $G$, it is also observed that for amplitudes $G<2 G_{c}$, the position of the peak [i.e., $\left.\omega^{*}(G)\right]$ increases toward higher frequencies, whereas for values $G>2 G_{c}$, the peak position shifts toward lower frequencies. It is not easy to give an exact cause for this behavior as both $G$ and $\omega$ are competing with each other in this region. Also, note that for
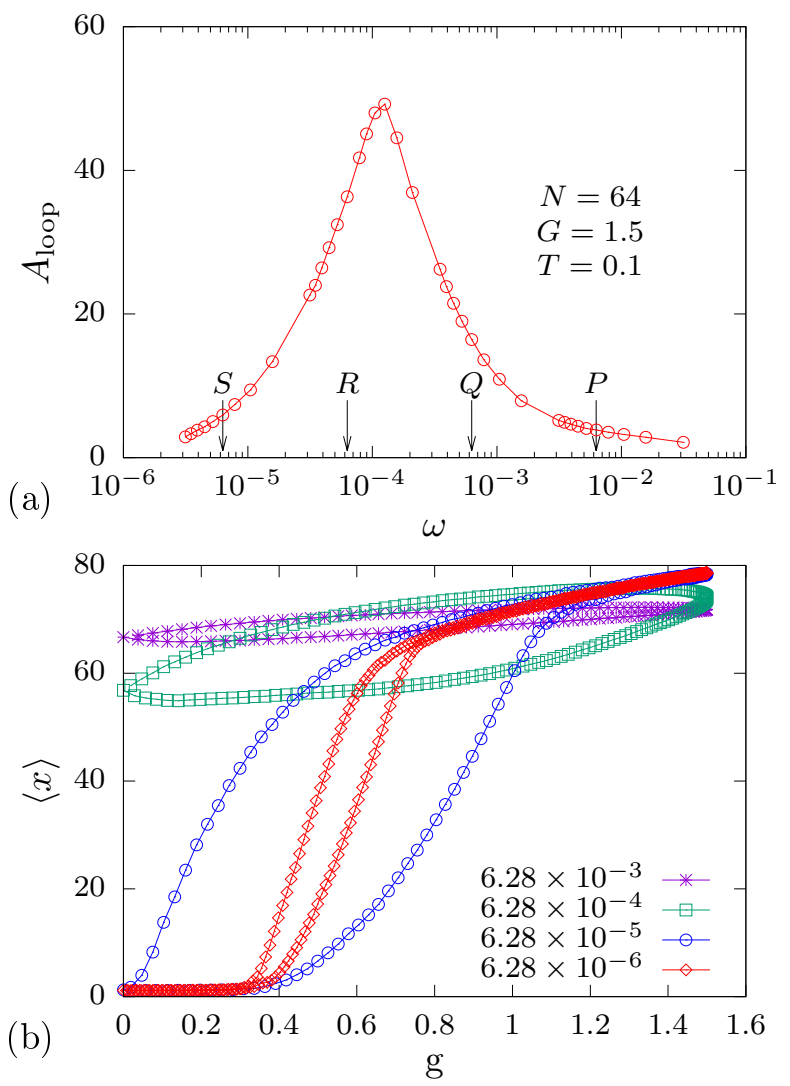

FIG. 4. (a) Area of hysteresis loop $A_{\text {loop }}$ as a function of frequency $\omega$, in a semilog scale, for the DNA of length $N=$ 64 and force amplitude $G=1.5$ at $T=0.1$. (b) Average extension $\langle x\rangle$ as a function of force $\mathrm{g}$ as various frequencies indicated in (a) by arrows. The line joining the points in these plots is just a guide for the eye.

$G<2 G_{c}$, the steady state of the DNA is a zipped configuration and it cannot be fully unzipped. Whereas, for $G>2 G_{c}$, the steady state of the DNA is an unzipped configuration. Consequently, the way the hysteresis loops are formed for the two cases are different and have different shapes 24. It is quite plausible that the dependence of maximum $A_{\text {loop }}$ on the frequency might be completely different in these two different regions. The figure also reveals that the height of the peak increases on increasing $G$ value. These observations are similar to the behavior seen for the $A_{\text {loop }}$ in MC simulations for the homopolymer DNA 24. There is one striking feature, the oscillatory behavior of $A_{\text {loop }}$ at higher frequencies for larger $G$ values, which was observed in MC simulations and explained as higher Rouse modes 24, is not observed with the parameters used in this study. However, the presence of such oscillatory behavior of $A_{\text {loop }}$ has been reported in LD simulations with different parameters [22]. This needs further exploration.

The loop area, $A_{\text {loop }}$ as a function of frequency $\omega$ for amplitude $G=1$ and 3, are plotted in Figs. 6(a) and 6(b), respectively, for the DNA of various chain lengths $N=16,32,64$ and 96 . The figure shows that, similar 


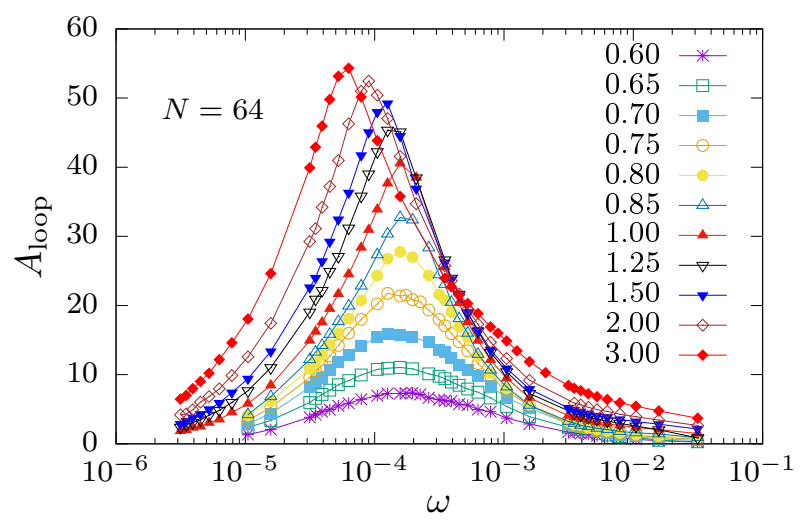

FIG. 5. Area of hysteresis loop $A_{\text {loop }}$ as a function of frequency $\omega$, in a semilog scale, for the DNA of length $N=64$ at various force amplitudes $G$. The line joining the points in these plots is just a guide for the eye.

to the MC simulation studies 24, the peak of the area curves shift toward the lower-frequency side on increasing the chain length. Furthermore, these plots also show that the maximum of the loop area increases with amplitude $G$. We use FSS of the form

$$
A_{\text {loop }}=N^{d} \mathcal{A}\left(\omega N^{z}\right),
$$

to obtain the behavior of $A_{\text {loop }}$ in the thermodynamic limit from finite-size chains. We obtain a nice collapse for exponents $d=1.00 \pm 0.05$ and $z=1.00 \pm 0.05$ for $G=1$ [Fig. 6(c)]. These exponent values are same as that obtained in MC simulation study [24]. However, for higher force amplitudes (e.g., $G=3$ ) we get a reasonable collapse for a slightly higher value $d=1.15 \pm 0.05$. Figures 6(c) and 6(d) show that there are strong finitesize effects and the curves for the smallest chain length $N=16$ considered in this study do not collapse perfectly on the scaling curve. However, the data for the higher chain lengths, $N=64$ and 96 , collapse perfectly on the scaling curve for $G=1$. In order to improve the quality of data collapse at force amplitude $G=3$, longer chain lengths need to be simulated. But, due to the higher computation cost [33], these simulations were not performed. The exponents $d=1$ and $z=1$ show that the loop area scales as $A_{\text {loop }} \sim 1 / \omega$ in the high-frequency regime.

To obtain the behavior of $A_{\text {loop }}$ at lower-frequency regime, we have plotted in Fig. 7(a) (in a semilog scale) the loop area as a function of $\omega^{\beta}\left(G-G_{c}\right)^{\alpha}$, where $G_{c}$ is the critical force for the static force case, obtained using LD simulations for the DNA of length $N=64$ at various force amplitudes $G=1.0,1.25,1.5,2.0$, and 3.0 at temperature $T=0.1$. We obtain an excellent data collapse for exponents $\alpha=1.08 \pm 0.03$ and $\beta=1.25 \pm 0.03$ and critical force $G_{c}(T=0.1)=0.45$ obtained for the static force case in the previous section (Eq. (7)). The exponents and the errors in them are estimated by minimizing the variance obtained from $A_{\text {loop }}$ curves for various $G$ values integrated over a decade in frequency [36]. In Fig. 7(b), the collapse obtained for $A_{\text {loop }}$ curves for chain length $N=32$ at $T=0.2$ for three different force amplitudes $G=1.0,1.5$, and 2.0 are plotted with $G_{c}(T=0.2)=0.25$ and $\alpha=1.09 \pm 0.03$ and $\beta=1.24 \pm 0.02$. The quality of the collapse indicates that the exponent values $\alpha$ and $\beta$ are independent of temperature used. Furthermore, these exponent values are similar to that obtained in previous studies using MC simulations of a DSAW model of the homopolymer DNA at $T=1$ [24, and the double stranded block copolymer DNA at $T=4$ [26]. In the homopolymer DNA study, the $A_{\text {loop }}$ was plotted against $G^{\alpha} \omega^{\beta}$. To check the quality of collapse with newer scaled function, we have plotted the $A_{\text {loop }}$ data, for the chain length $N=512$, obtained in Ref. 24] as a function of $\omega^{\beta}\left(G-G_{c}\right)^{\alpha}$ with $G_{c}(T=1)=0.678$ and $\alpha=0.99 \pm 0.03$ and $\beta=1.25 \pm 0.03$. The quality of the plot shown in Fig. 7(c) is found to be even better than the plot shown with function $G^{\alpha} \omega^{\beta}$ in Ref. [24].

We can use the behavior of $A_{\text {loop }}$ in low- and highfrequency regimes to obtain the scaling function $\mathcal{G}(\omega)$. At lower frequencies (i.e., $\omega \rightarrow 0$ ), we observed that, for large $N$, the $A_{\text {loop }}$ scales as $G^{\alpha} \omega^{\beta}$, while at higher frequencies (i.e., $\omega \rightarrow \infty$ ), $A_{\text {loop }} \sim 1 / \omega$ [from Eq. (10)]. These requirements are satisfied by the scaling function

$$
\mathcal{G}(\omega)=\frac{B G^{\alpha} \omega^{\beta}}{\omega^{d+\beta}+C^{2}}
$$

with $B$ and $C$ as the fitting parameters. The scaling function, $\mathcal{G}(\omega)$, for $G=1$ with exponent $d=1$, and parameters $B=0.01$ and $C=0.005$ obtained by data fitting, is plotted in Fig. 6(c) by a solid line. The function fits the data extremely well in the frequency range extended over more than four decades. We have also plotted the same scaling function for $G=3$, with exponent $d=1.15$ for parameters $B=0.001$ and $C=0.002$ in Fig. 6(d). Although the scaling function $\mathcal{G}(\omega)$ fits reasonably well in the lower-frequency regime, it however deviates with the scaled data at higher frequencies. From MC simulation studies [24], we know that for $G=3, A_{\text {loop }}$ exhibits an oscillatory behavior in the higher-frequency regime (visible only for longer chain lengths) and the above scaling form is not suitable. Since the maximum chain length simulated in this study, is still 5 times lesser than that simulated in Ref. 24], we do not see the oscillatory behavior of $A_{\text {loop }}$ at higher frequencies. The deviation of the simulation data from the scaled curve $\mathcal{G}(\omega)$ for $G=3$ at higher-frequency regime in Fig. 7(d) may be due to this very reason.

\section{CONCLUSIONS}

We study the unzipping of a dsDNA subjected to a periodic force with amplitude $G$ and frequency $\omega$ using extensive LD simulations on longer DNA chains, having up to 192 monomers with $N=96$ base pairs, that are six times longer than previous LD simulation studies [18 22$]$. 

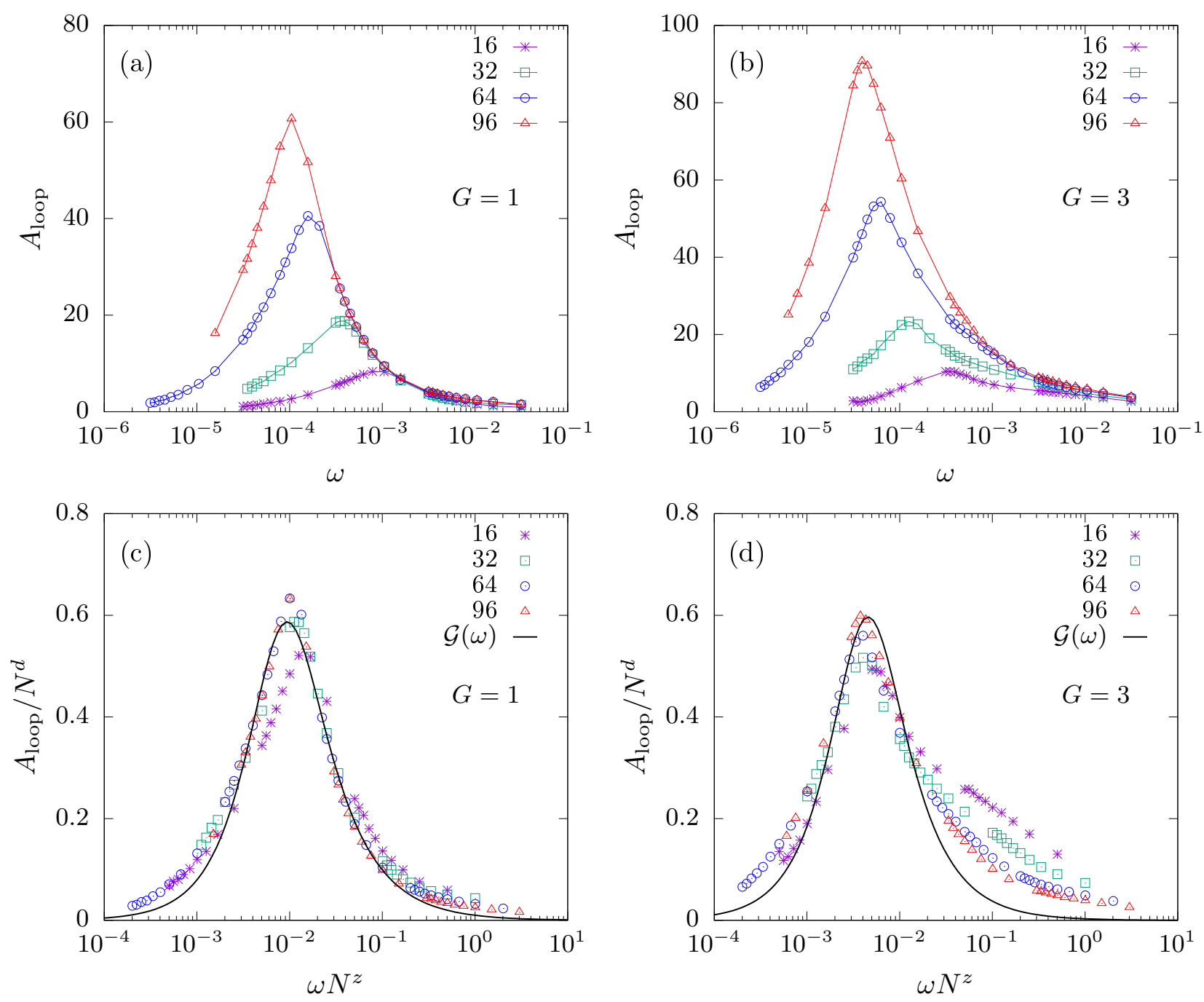

FIG. 6. Area of hysteresis loop $A_{\text {loop }}$ as a function of frequency $\omega$, in a semilog scale, for DNA of lengths $N=16,32,64$, and 96 for force amplitudes (a) $G=1$ and (b) $G=3$. Scaled loop area, $A_{\text {loop }} / N^{d}$, plotted against $\omega N^{z}$ showing a nice collapse for exponents (c) $d=1.00 \pm 0.05$ and $z=1.00 \pm 0.05$ for $G=1$, and (d) $d=1.15 \pm 0.05$ and $z=1.00 \pm 0.05$ for $G=3$. The line joining the points in these plots is just a guide for the eye.

We first study the static force case and obtain the equilibrium average separation between the strands of the DNA, $\langle x(\mathrm{G})\rangle$, as a function of force $\mathrm{G}$ at two different temperatures $(T=0.1$ and $T=0.2)$. Using the FSS of force-distance isotherms $\langle x(\mathrm{G})\rangle$ for various chain lengths $N=64,96,128,192$, and 256 , we obtain the dimensionless critical force $G_{c}(T=0.1)=0.45 \pm 0.05$ at $T=0.1$ and $G_{c}(T=0.2)=0.25 \pm 0.05$ at $T=0.2$, needed to unzip the DNA in the thermodynamic limit $N \rightarrow \infty$. The FSS reveals that the scaled average separation between the strands, $\langle x\rangle / N$, has a jump discontinuity at $G_{c}$ implying a first-order nature of the phase transition similar to the DSAW model studied earlier 2, 5, 77. We also obtained the melting temperature $T_{M}=0.30 \pm 0.01$ for the model. The melting of DNA is a continuous transition in our model. When the DNA is subjected to a peri- odic force, the average separation between the strands $\langle x(\mathrm{~g})\rangle$, when plotted against $\mathrm{g}$, shows hysteresis whose area, $A_{\text {loop }}$, depends on the amplitude $G$ and the frequency $\omega$ of the oscillating force. On decreasing the frequency, the loop area first increases from zero, reaches a maximum value at some frequency $\omega^{*}(G)$, which depends on the amplitude $G$, and then decreases to zero again at lower frequencies. The FFS scaling of Eq. (10) shows that, in the thermodynamic limit, the loop area scales as $A_{\text {loop }} \sim 1 / \omega$ in the higher-frequency regime. In contrast, the loop area which scales as $A_{\text {loop }} \sim\left(G-G_{c}\right)^{\alpha} \omega^{\beta}$ is found to have exponent values $\alpha \approx 1$ and $\beta \approx 1.25$, The exponent values, which are found to be temperature independent, are same as that obtained in earlier unzipping studies of homopolymer DNA [24] and block copolymer DNA [26] by a periodic force on a DSAW model using 

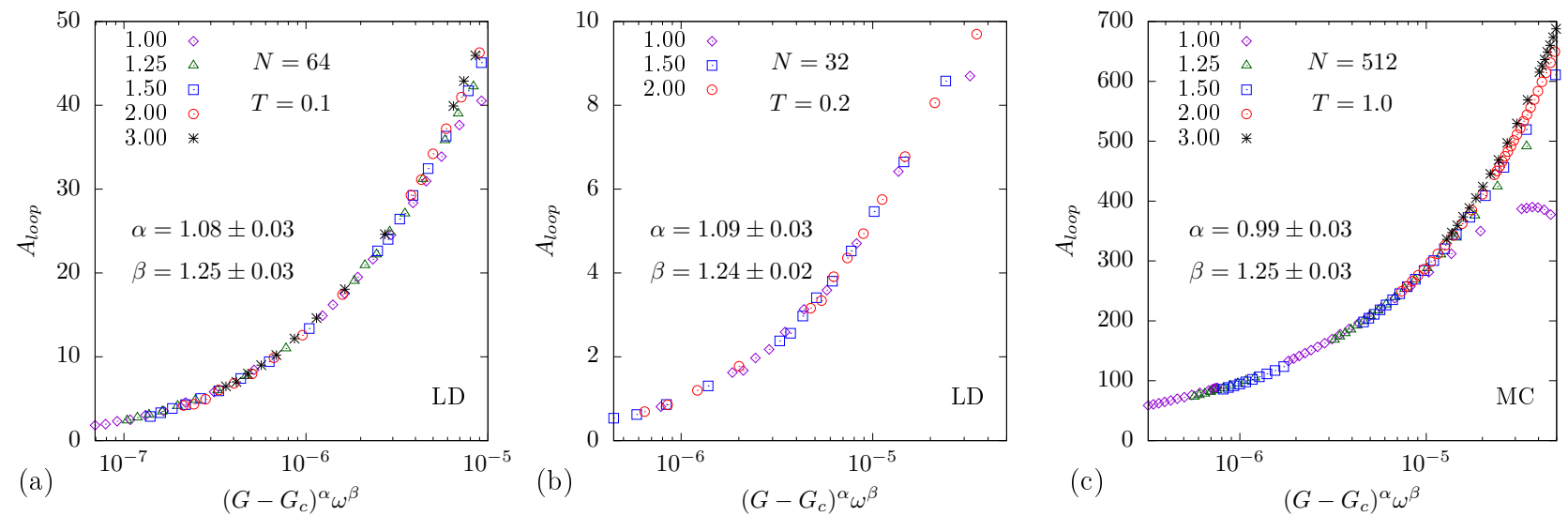

FIG. 7. $A_{\text {loop }}$ as a function of $\left(G-G_{c}\right)^{\alpha} \omega^{\beta}$ for various force amplitudes obtained (a) using LD simulations for the DNA of length $N=64$ at $T=0.1$ with $G_{c}(T=0.1)=0.45 \pm 0.05$ with exponents $\alpha=1.08 \pm 0.03$ and $\beta=1.25 \pm 0.03$, (b) using LD simulations for the DNA of length $N=32$ at $T=0.2$ with $G_{c}(T=0.2)=0.25 \pm 0.05$ with exponents $\alpha=1.09 \pm 0.03$ and $\beta=1.24 \pm 0.02$, (c) using MC simulations of the DSAW model of DNA of length $N=512$ at $T=1$ with $G_{c}(T=1)=0.678$ with exponents $\alpha=0.99 \pm 0.03$ and $\beta=1.25 \pm 0.03$, as obtained in Ref. [24].

MC simulations at two different temperatures. The fact that we have obtained the same values for the exponents, $\alpha$ and $\beta$, at various temperatures for two different problems, i.e., homopolymer DNA and block copolymer DNA, where the former is studied by two different methods, MC and LD simulations of longer chain lengths at different temperatures strongly indicates that $\alpha=1.0$ and $\beta=1.25$ are the true scaling exponents for the DNA unzipping problem that quantify the decrease of $A_{\text {loop }}$ to zero at low frequencies at all temperatures. Single molecule manipulation experiments can shed more light on these scaling exponents.

\section{ACKNOWLEDGEMENTS}

I thank S. M. Bhattacharjee, A. Chaudhuri and R. Yadav for their comments on the manuscript. I thank A. Chaudhuri for allowing me to use his computational resources.
[1] J. D. Watson, T. A. Baker, S. P. Bell, A. Gann, M. Levine, and R. Losick, Molecular Biology of the Gene, 5th ed. (Pearson/Benjamin Cummings, Singapore, 2003).

[2] S. M. Bhattacharjee, J. Phys. A: Math. Gen 33, L423 (2000).

[3] D. K. Lubensky and D. R. Nelson, Phys. Rev. Lett. 85, 1572 (2000).

[4] K. L. Sebastian, Phys. Rev. E 62, 1128 (2000)

[5] D. Marenduzzo, A. Trovato, and A. Maritan, Physical Review E 64, 031901 (2001)

[6] D. Marenduzzo, S. M. Bhattacharjee, A. Maritan, E. Orlandini, and F. Seno, Physical Review Letters 88, 028102 (2002)

[7] R. Kapri, S. M. Bhattacharjee, and F. Seno, Physical Review Letters 93, 248102 (2004).

[8] S. Kumar and M. S. Li, Physics Reports 486, 1 (2010)

[9] U. Bockelmann, P. Thomen, B. Essevaz-Roulet, V. Viasnoff, and F. Heslot, Biophysical Journal 82, 1537 (2002).

[10] C. Danilowicz, V. W. Coljee, C. Bouzigues, D. K. Lubensky, D. R. Nelson, and M. Prentiss, Proc. Natl. Acad. Sci. U.S.A. 100, 1694 (2003).

[11] C. Danilowicz, Y. Kafri, R. S. Conroy, V. W. Coljee, J. Weeks, and M. Prentiss, Physical Review Letters 93, 078101 (2004)

[12] F. Ritort, J. Phys. Condens. Matter 18, R531 (2006)
[13] K. Hatch, C. Danilowicz, V. Coljee, and M. Prentiss, Physical Review E 75, 051908 (2007).

[14] R. W. Friddle, P. Podsiadlo, A. B. Artyukhin, and A. Noy, J. Phys. Chem. C 112, 4986 (2008).

[15] Z. Tshiprut and M. Urbakh, J. Chem. Phys. 130, 084703 (2009).

[16] P. T. X. Li, C. Bustamante, and I. Tinoco, Proc. Natl. Acad. Sci. U.S.A. 104, 7039 (2007).

[17] A. Yasunaga, Y. Murad, and I. T. S. Li, Phys. Biol. 17, 011001 (2019)

[18] S. Kumar and G. Mishra, Physical Review Letters 110, 258102 (2013)

[19] G. Mishra, P. Sadhukhan, S. M. Bhattacharjee, and S. Kumar, Physical Review E 87, 022718 (2013).

[20] R. K. Mishra, G. Mishra, D. Giri, and S. Kumar, J Chem Phys 138, 244905 (2013)

[21] S. Kumar, R. Kumar, and W. Janke, Phys. Rev. E 93, 010402(R) (2016)

[22] T. Pal and S. Kumar, Eurphys. Lett. 121, 18001 (2018)

[23] R. Kapri, Physical Review E 86, 041906 (2012).

[24] R. Kapri, Phys. Rev. E 90, 062719 (2014)

[25] M. S. Kalyan and R. Kapri, J. Chem. Phys. 150, 224903 (2019)

[26] R. K. Yadav and R. Kapri, Phys. Rev. E 103, 012413 (2021) 
[27] R. Kapri and S. M. Bhattacharjee, Journal of Physics: Condensed Matter 18, 215 (2006)

[28] R. Kapri and S. M. Bhattacharjee, Physical Review Letters 98, 098101 (2007)

[29] R. Kapri and S. M. Bhattacharjee, Europhys. Lett. 83, $68002(2008)$

[30] G. S. Grest and K. Kremer, Phys. Rev. A 33, 3628 (1986).

[31] S. Plimpton, J. Comp. Phys. 117, 1 (1995).

[32] B. K. Chakrabarti and M. Acharyya, Rev. Mod. Phys. 71, 847 (1999)

[33] At a given force amplitude $G$ and frequency $\omega$, the computation time increases linearly with the system size $N$. For a given system size $N$, the computation time increases linearly by the same order of magnitude by which the frequency $\omega$ is decreased. For example, on Intel Xeon Processor E5-2650 v4 running at 2.20 GHz, the total com- putation cost for obtaining $A_{\text {loop }}$ (from 220 cycles) at force amplitude $G=3$ and frequency $\omega=6.28 \times 10^{-6}$ were 10.5 and 14.8 days for chain lengths $N=64$ and $N=96$, respectively. On decreasing the frequency to $\omega=3.14 \times 10^{-6}$, keeping the force amplitude same, the computation cost became 19.5 days for $N=64$. At this frequency, it would have required approximately 29 days for $N=96$.

[34] M. Galassi et al., Gnu Scientific Library Reference Manual, 3rd ed. (Network Theory Ltd., Bristol, UK, 2009).

[35] M. Rubinstein and R. H. Colby, Polymer Physics, (Oxford University Press, New York, 2003).

[36] M. E. J. Newman and G. T. Barkema, Monte Carlo Methods in Statistical Physics, (Clarendon Press, Oxford, 1999). 